\title{
Dynamization-Posterior Lumbar Interbody Fusion for Hemodialysis-Related Spondyloarthropathy: Evaluation of the Radiographic Outcomes and Reoperation Rate within 2 Years Postoperatively
}

\author{
Taiki Yasukawa ${ }^{1,2}$, Junichi Ohya ${ }^{1}$, Naohiro Kawamura ${ }^{1}$, Yuichi Yoshida ${ }^{1}$, Yuki Onishi ${ }^{1}$, Kazuhiro Kohata ${ }^{1}$, \\ Yohei Kakuta ${ }^{1}$, Satoshi Nagatani ${ }^{1}$, Yoshifumi Kudo ${ }^{2}$, Toshiyuki Shirahata ${ }^{2}$, Junichi Kunogi ${ }^{1}$ \\ ${ }^{1}$ Department of Spine and Orthopedic Surgery, Japanese Red Cross Medical Center, Tokyo, Japan \\ ${ }^{2}$ Department of Orthopedic Surgery, Showa University School of Medicine, Tokyo, Japan
}

\begin{abstract}
Study Design: Clinical case series.
Purpose: This study aimed to report dynamization-posterior lumbar interbody fusion (PLIF), our surgical treatment for hemodialysisrelated spondyloarthropathy (HSA), and investigate patients' postoperative course within 2 years.

Overview of Literature: HSA often requires lumbar fusion surgery. Conventional PLIF for HSA may cause progressive destructive changes in the vertebral endplate, leading to progressive cage subsidence, pedicle screw loosening, and pseudoarthrosis. A dynamic stabilization system might be effective in patients with a poor bone quality. Thus, we performed "dynamization-PLIF" in hemodialysis patients with destructive vertebral endplate changes.

Methods: We retrospectively examined patients with HSA who underwent dynamization-PLIF at our hospital between April 2010 and March 2018. The radiographic measurements included lumbar lordosis and local lordosis in the fused segment. The evaluation points were before surgery, immediately after surgery, 1 year after surgery, and 2 years after surgery. The preoperative and postoperative radiographic findings were compared using a paired t-test. A p-value of less than 0.05 was considered significant.

Results: We included 50 patients (28 males, 22 females). Lumbar lordosis and local lordosis were significantly improved through dynamization-PLIF (lumbar lordosis, $28.4^{\circ}-35.5^{\circ}$; local lordosis, $2.7^{\circ}-12.8^{\circ} ; p<0.01$ ). The mean local lordosis was maintained throughout the postoperative course at 1 - and 2-year follow-up $\left(12.9^{\circ}-12.8^{\circ}, p=0.89\right.$ and $12.9^{\circ}-11.8^{\circ}, p=0.07$, respectively). Solid fusion was achieved in 59 (89\%) of 66 fused segments. Solid fusion of all fixed segments was achieved in 42 cases (84\%). Within 2 years postoperatively, only six cases (12\%) were reoperated (two, surgical debridement for surgical site infection; two, reoperation for pedicle screw loosening; one, laminectomy for epidural hematoma; one, additional fusion for adjacent segment disease).

Conclusions: Dynamization-PLIF showed local lordosis improvement, a high solid fusion rate, and a low reoperation rate within 2 years of follow-up.
\end{abstract}

Keywords: Hemodialysis; Destructive spondyloarthropathy; Posterior lumbar interbody fusion; Dynamic stabilization; Dynamization

Received Jul 31, 2021; Revised Oct 5, 2021; Accepted Oct 5, 2021

Corresponding author: Taiki Yasukawa

Department of Spine and Orthopedic Surgery, Japanese Red Cross Medical Center, 4-1-22, Hiroo, Shibuya-ku, Tokyo, Japan

Tel: +81-3-3400-1311, Fax: +81-3-3400-0193, E-mail: taiki.yasu0123@gmail.com 


\section{Introduction}

Hemodialysis technology has advanced in recent years, extending the life expectancy of patients undergoing hemodialysis $[1,2]$. However, the prevalence of hemodialysis-related spondyloarthropathy (HSA) has increased. Among the types of HSA, destructive spondyloarthropathy (DSA), which was first described by Kuntz et al. [3] in 1984, is the most serious musculoskeletal complication in long-term dialysis. The incidence of DSA among patients receiving hemodialysis is $18 \%-20 \%[4,5]$. DSA development is reportedly related to age and hemodialysis duration, and it is more commonly identified at the cervical spine than at the lumbar spine [5]. In DSA, amyloid accumulates at the enthesis of collagen fibers, facet joint, annulus fibrosus, and capsule, leading to spinal instability $[6,7]$. DSA also leads to destructive changes, including destructive kyphosis, vertebral endplate erosion, spondylolisthesis, extradural amyloid deposit, and hypertrophied ligamentum flavum, causing back pain, spinal deformity, and neurological symptoms. Lumbar spine surgery is often considered for HSA treatment, but the rate of reoperation after decompression surgery is relatively high (12.5\%) [8].

Patients with high instability caused by destructive changes frequently require lumbar fusion surgery. In particular, conventional posterior lumbar interbody fusion (PLIF) procedure has provided good short-term results in patients with HSA [2]. However, progressive destructive changes in the vertebral endplate can lead to progressive cage subsidence and pedicle screw loosening (Fig. 1). Consequently, a fair number of cases of pseudarthrosis have been reported, with $21.5 \%-40.0 \%$ carrying reoperation risk [9-12].

Dynamic stabilization systems achieve mobile stabilization by using pedicle screws and have been reported useful for the surgical treatment of lumbar discopathy, spondylolisthesis, and degenerative scoliosis [13-17]. We hypothesized that a dynamic stabilization system is effective in patients with a poor bone quality. Thus, we have applied this approach to lumbar interbody fusion for patients with hemodialysis as "dynamization-PLIF." In this study, we aimed to report our surgical treatment by dynamization-PLIF for patients with HSA and assess the postoperative course within 2 years of follow-up.

\section{Materials and Methods}

This study was approved by the institutional review board (IRB) of the Japanese Red Cross Medical Center (IRB approval no., 748). The requirement for informed consent from individual patients was omitted because of the retrospective design of this study. We retrospectively reviewed the medical records of patients with HSA who underwent dynamization-PLIF at our hospital between April 2010 and March 2018. Dynamization-PLIF was specifically
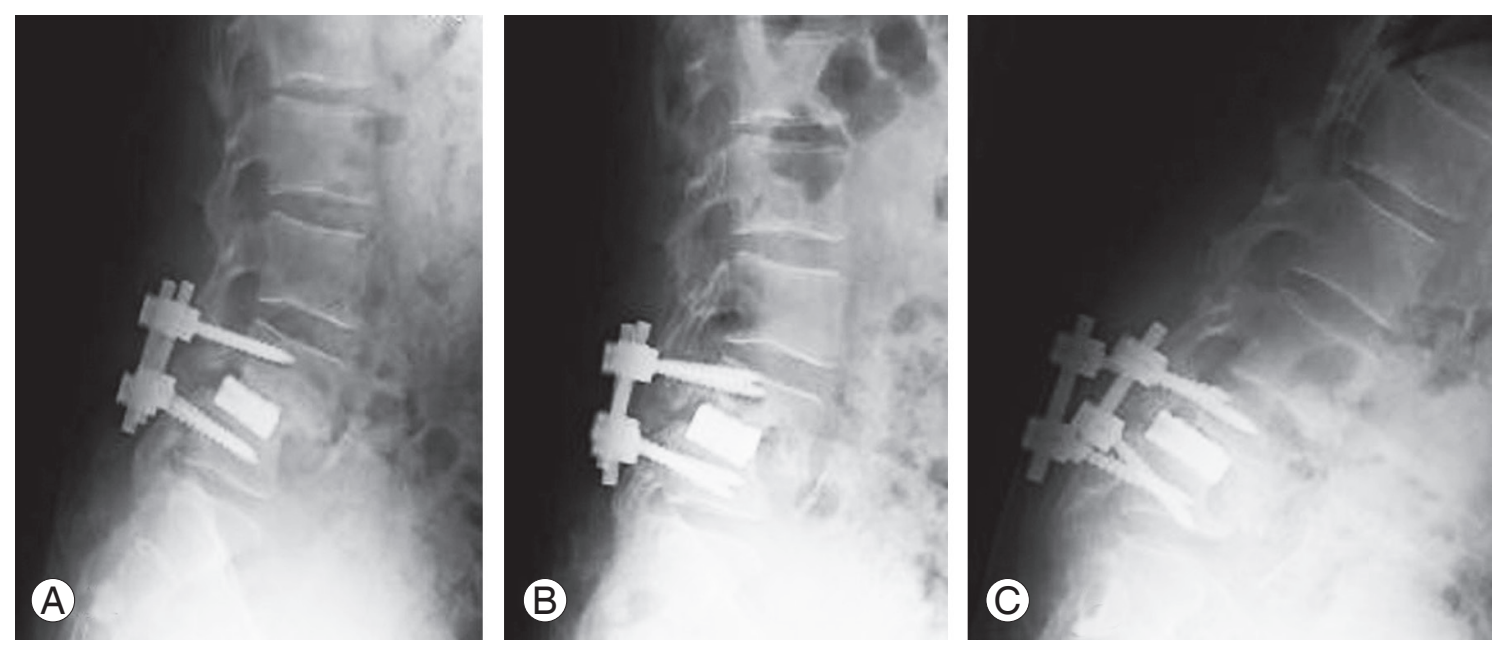

Fig. 1. (A) Postoperative radiograph of the lumbar spine after conventional posterior lumbar interbody fusion for a hemodialysis-related spondyloarthropathy patient. (B) Postoperative lateral radiograph of the lumbar spine three months after surgery. (C) Postoperative radiograph of the lumbar spine six months after surgery showing that progressive destructive changes in the vertebral endplate have led to progressive cage subsidence and pedicle screw loosening. 


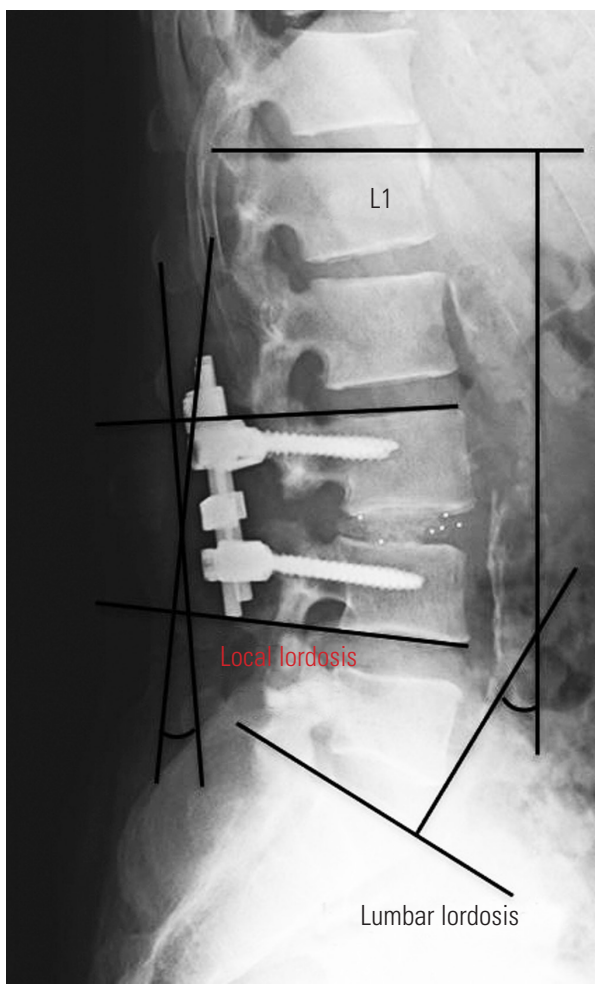

Fig. 2. Measurements on radiograph of lumbar lordosis and local lordosis in the fused segment.

performed to patients with destructive changes in the vertebral endplates. For those with spinal canal stenosis associated with HSA without instability, we performed decompression alone. We excluded patients who died or whose radiographic findings could not be evaluated within 2 years after surgery. The radiographic measurements included lumbar lordosis and local lordosis in the fused segment. Local lordosis was measured as the angle between the proximal and distal vertebral endplates of the fused segment (Fig. 2). The evaluation points were before surgery, immediately after surgery, 1 year after surgery, and 2 years after surgery. We assessed whether the fixed segment achieved solid fusion or not at 2 years postoperatively. We also investigated reoperations within 2 years after surgery and checked the causes and contents.

\section{Dynamization-posterior lumbar interbody fusion surgical procedure}

Dynamization-PLIF is our modified PLIF procedure in which the instrumented vertebra is allowed to partially move before bone union. In the final fixation of the instruments after the conventional PLIF procedure, the rods are

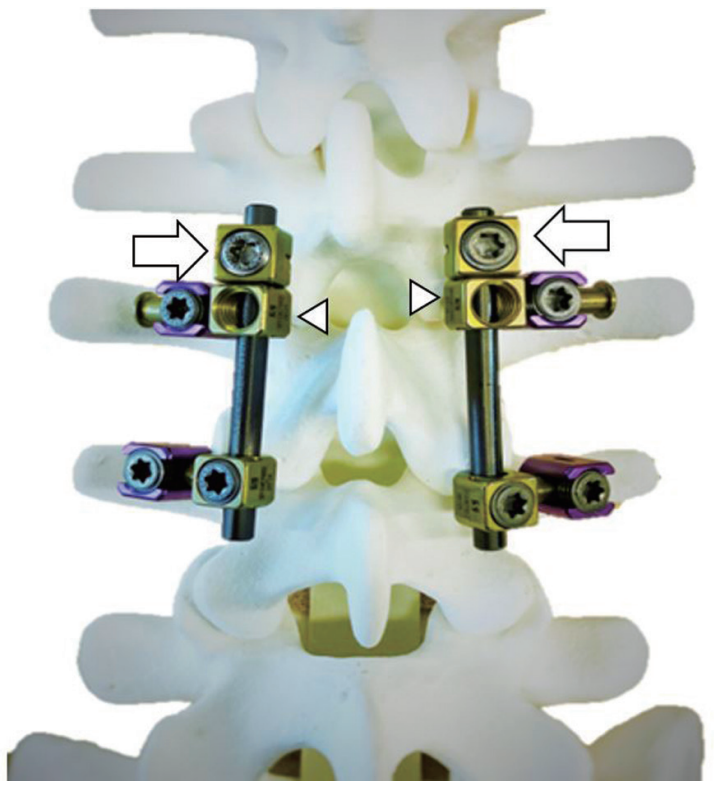

Fig. 3. Model of the dynamization-posterior lumbar interbody fusion procedure. The implant system used was the MYKRES Spinal System (Teijin Nakashima Medical, Tokyo, Japan). The rods are placed with lateral connectors onto the pedicle screws. Although one side of the connector is fixed by a set-screw as usual, the other side is set free (arrowheads), allowing the movement of relevant vertebra. A stopper (arrows) is placed next to each free connector to block opening of the intervertebral space.

placed with lateral connectors onto the pedicle screws (Fig. 3). Although one side of the connector is fixed by a set screw as usual, the other side is left free (arrowheads), allowing the relevant vertebrae to move. The opening of the intervertebral space is blocked by placing a stopper (arrow) next to each free connector. This system allows the mobile instrumented vertebra to slide along the rod with interbody space shortening while maintaining lumbar lordosis. Considering that the interbody cage is allowed to sink, the bilateral foraminal space should also be decompressed to allow for later narrowing $[18,19]$. The implant system used was the MYKRES Spinal System (Teijin Nakashima Medical, Tokyo, Japan).

\section{Outcome}

Solid fusion was defined using the following criteria: less than $3^{\circ}$ vertebral motion angle in forward and backward bending, radiological bony bridge formation in the fusion area, and no gap between the cage and the vertebral body [20-22]. Failure to meet any of these criteria indicated nonfusion. Solid fusion was assessed by one spine surgeon using only radiographs without computed tomography. 
Information on reoperation was reviewed in the medical records.

\section{Statistical analyses}

The values are presented as the mean \pm standard deviation for continuous variables or number (percentage) for categorical variables. The preoperative and postoperative radiographic findings were compared using a paired $t$-test. A $p$-value of less than 0.05 was considered significant.

\section{Results}

Within 2 years of follow-up, two patients (3.8\%) died. We included 50 patients ( 28 males, 22 females). The mean patient age was $64.7 \pm 6.4$ years (range, $49-82$ years), and the mean hemodialysis duration was $16.4 \pm 9.9$ years (range, 1-36 years). The surgical fusion level was one in 35 cases, two in 14 cases, and three in one case. The operative time was $217.6 \pm 53.5$ minutes, and the estimated blood loss was $461.7 \pm 338.8 \mathrm{~mL}$ (Table 1).

Lumbar lordosis and local lordosis were significantly improved through dynamization-PLIF (lumbar lordosis, $28.4^{\circ}-35.5^{\circ}$; local lordosis, $\left.2.7^{\circ}-12.8^{\circ} ; p<0.01\right)$. At 1 and 2-year follow-up, lumbar lordosis was significantly improved compared with that immediately after surgery $\left(35.6^{\circ}-39.4^{\circ}, p<0.01\right.$ and $35.6^{\circ}-38.4^{\circ}, p<0.05$, respectively)

Table 1. Patients' characteristics

\begin{tabular}{lc}
\hline Characteristic & \multicolumn{1}{c}{ Value } \\
\hline No. of patients & 50 \\
\hline Mean age at the time of surgery (yr) & $64.7 \pm 6.4(49-82)$ \\
\hline Gender & \\
\hline Male & 28 \\
\hline Female & $16.4 \pm 9.9(3-36)$ \\
\hline Duration of hemodialysis at surgery (yr) & $48.9(24-109)$ \\
\hline Duration of follow-up (mo) & 35 \\
\hline No. of fused levels & 14 \\
\hline \multicolumn{1}{|c|}{ Level } & 1 \\
\hline 2 Levels & $217.6 \pm 53.5(143-335)$ \\
\hline 3 Levels & $461.7 \pm 339(30-1,770)$ \\
\hline Operation time (min) & \\
\hline Estimated blood loss (mL) & \\
\hline
\end{tabular}

Values are presented as number, mean \pm standard deviation (range), or median (range).
(Fig. 4), whereas local lordosis was maintained overall throughout the postoperative course $\left(12.9^{\circ}-12.8^{\circ}, p=0.89\right.$ and $12.9^{\circ}-11.8^{\circ}, p=0.07$, respectively).

Among the 66 fused segments, 59 (89\%) achieved solid fusion. Furthermore, 42 cases (84\%) achieved solid fusion of all fixed segments. Reoperation occurred in six patients within 2 years after surgery (two, surgical debridement for surgical site infection; two, reoperation for pedicle screw loosening; one, laminectomy for epidural hematoma; one, additional fusion for adjacent segment disease [ASD]). While the average duration of hemodialysis was $14.0 \pm 4.0$ years in those with complications, that in those without complications was $17 \pm 1.5$ years, indicating no significant differences between these two groups.

\section{Representative case presentation}

A 60-year-old man who had been on hemodialysis for 12 years complained of intermittent claudication and lower back pain. Preoperative radiographs of the lumbar spine demonstrated DSA, and magnetic resonance imaging revealed severe spinal stenosis (Fig. 5A, B). After undergoing dynamization-PLIF at L3-L4 (Fig. 5C), his leg pain subsided immediately. At 2-year follow-up, postoperative radiographs showed interbody space shortening caused by cage sinking, as indicated by the gap between the stopper and lateral connector (Fig. 5D, arrow). No pedicle screw loosening was noted, and solid fusion was achieved.

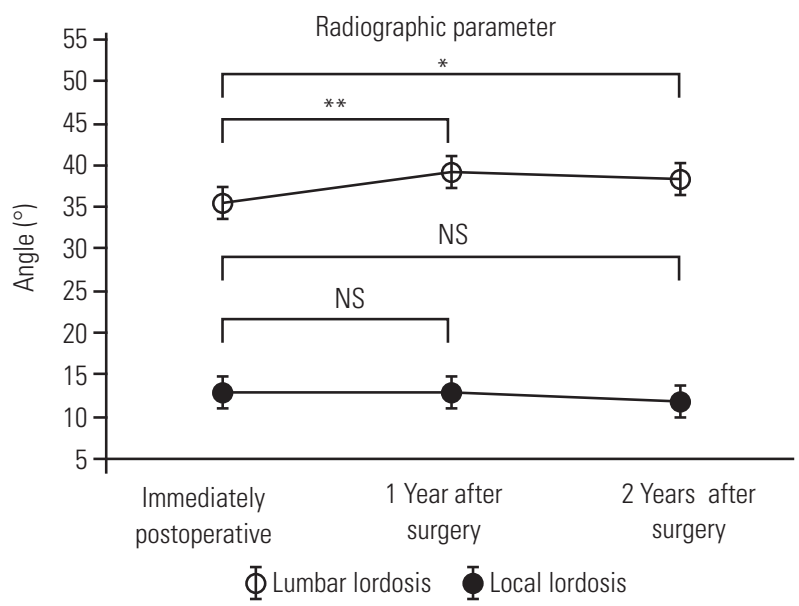

Fig. 4. Postoperative course of lumbar lordosis and local lordosis of the fused segment. Lumbar lordosis at 1- and 2-year follow-up had improved significantly compared to that immediately after surgery. Local lordosis did not change significantly. NS, not significant. ${ }^{*} p<0.05 .{ }^{* *} p<0.01$. 

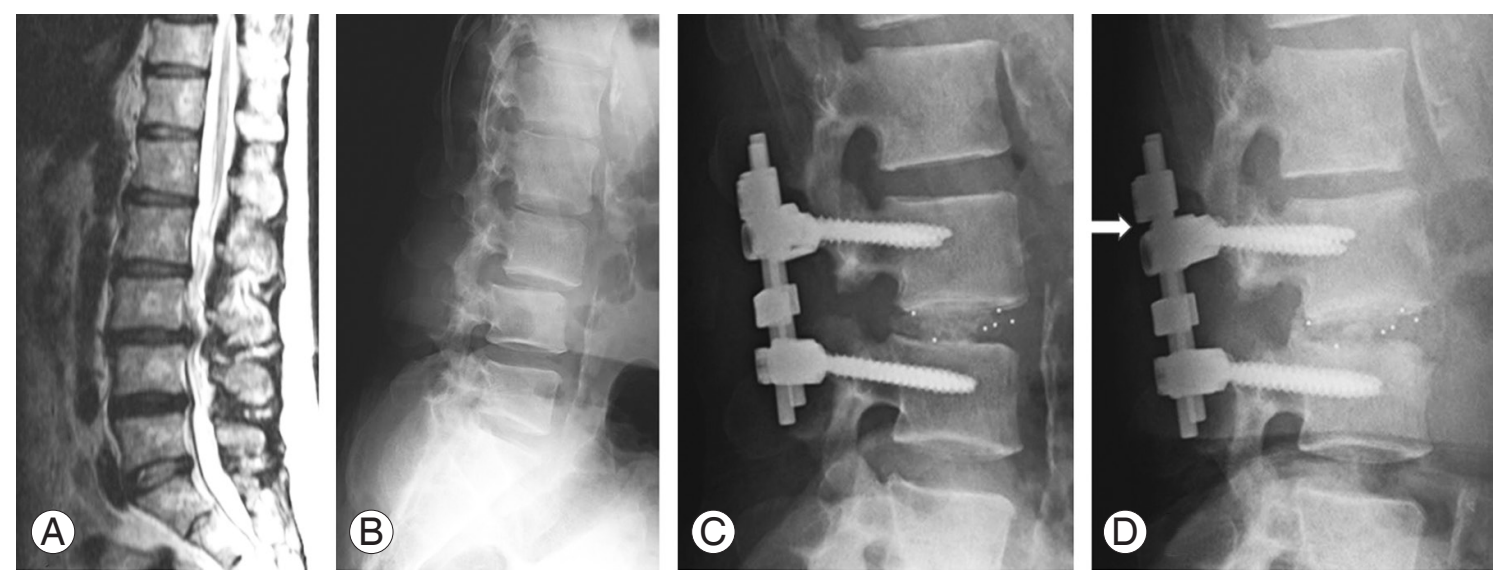

Fig. 5. Representative case presentation. (A) Magnetic resonance imaging showing canal stenosis at $L 3 / 4$. (B) Preoperative lateral radiograph showing degenerative spondylolisthesis at L3/4. (C) Postoperative lateral radiograph showing dynamization-posterior lumbar interbody fusion at L3/4. (D) Postoperative lateral radiograph after 2 years showing bone union with maintained local lordosis. A gap appeared between the stopper and connector (arrow)

\section{Discussion}

HSA is a general term for spondylolisthesis resulting primarily from amyloidosis. Among the HSAs, DSA is the most serious musculoskeletal complication for long-term dialysis. In a cross-sectional study, $20 \%$ of patients with hemodialysis suffered from DSA [4], which was associated with age and hemodialysis duration. Although many HSA cases require spine surgery to treat spinal canal stenosis with instability, the rates of perioperative complications and mortality following spinal surgery are reportedly high. Chikuda et al. [23] compared the risk for perioperative complications between patients with hemodialysis who underwent spinal surgery and those without hemodialysis. They concluded that the hemodialysis group had a tenfold higher risk of death than the nonhemodialysis group and that DSA increased the complication rates [23]. In addition, high rates of surgery-related complications, such as implant failure, pseudoarthrosis, and ASD, are a crucial problem in patients undergoing hemodialysis [2,9$12]$. Thus, the problems associated with lumbar fusion surgery for patients with hemodialysis include mechanical surgery-related complications as well as high mortality rate. One potential reason for the high rates of mechanical surgery-related complications would be interbody cage sinking caused by disruption of the fragile vertebral endplates with amyloid deposition. Collapse of the anterior fixation element creates a mismatch with the posterior fixation of elements, such as pedicle screws and rods, causing the decrease in fixation force until bone union and subsequent pseudoarthrosis. Conventional PLIF for patients with hemodialysis remains a challenging operation.

Lumbar discopathy, spondylolisthesis, and degenerative scoliosis benefit from dynamic stabilization [13-17]. Theoretically, the concept of dynamic stabilization involves intervertebral mobility maintenance, thereby reducing the strain on posterior elements, such as adjacent disks and facets, and preventing ASD development. Consequently, dynamic stabilization systems reduce the occurrence of adjacent segment degeneration at a short-term follow-up [24]. However, some studies with long-term observation reported that the segment of the lumbar spine stabilized by the dynamic stabilization system lost its mobility and fused at the final follow-up $[25,26]$. In this context, we developed a dynamic stabilization system for fusion surgery, targeting patients with a low bone density and quality who are at risk for implant failure.

Moreover, dynamic stabilization showed favorable outcomes in older patients with degenerative spondylolisthesis, with better fusion rates in fusion surgery using the dynamic stabilization system than in conventional fusion systems [27]. This finding supported our own system. Therefore, we started to apply dynamic stabilization to patients with HSA cases who were likely to develop cage sinking; the approach has been termed as "dynamizationPLIF." In hemodialysis cases, one potential reason for the high rates of mechanical surgery-related complications is interbody cage sinking caused by disruption of the fragile vertebral endplates with erosive amyloid deposition. Col- 
lapse of the anterior element results in mismatch with the posterior fixed elements, such as pedicle screws and rods, leading to pedicle screw loosening and subsequent pseudoarthrosis and kyphotic deformity (Fig. 6) [28]. Nevertheless, the mismatch between the vertebral body and posterior elements can be resolved through dynamic stabilization by sliding the posterior elements without losing lordosis and without holding the force of pedicle screws until bone fusion. This system helped preserve the postoperative lumbar lordosis and local lordosis in pa-

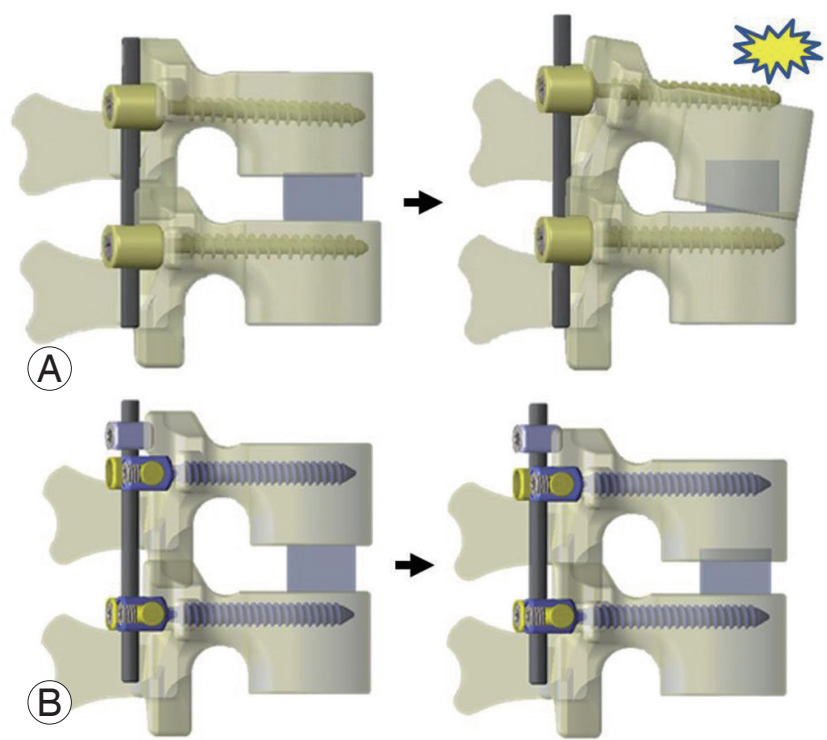

Fig. 6. The illustration of conventional posterior lumbar interbody fusion (PLIF) procedure and dynamization-PLIF procedure reproduced from [28] with permission. (A) Model of the conventional PLIF procedure. When the anterior element is shortened due to cage subsidence and pedicle screw loosening, it causes local kyphosis. (B) Model of the dynamization-PLIF procedure. As the posterior element of the subsiding vertebra is allowed to slide along the rods, sagittal alignment is preserved. tients with a poor bone quality.

High rates of reoperation for surgery-related complications, such as implant failure and pseudoarthrosis, are also crucial problems in patients with hemodialysis. Generally, lumbar fusion surgery for HSA carries a high risk for implant failure because of a poor bone quality. Yamada et al. [11] reported that $21 \%$ of patients with hemodialysis who underwent lumbar fusion surgery required revision surgery, with a mean follow-up of 3.2 years. Hori et al. [12] reported a matched cohort study of lumbar spinal surgery in patients with and without hemodialysis with a mean follow-up of 2.4 years and found that $37.5 \%$ of those with hemodialysis who underwent lumbar fusion surgery required reoperation for implant failure. In the present study, the revision rate was relatively low, being $6 \%$ during the 2-year follow-up. Maintaining lordosis through the postoperative course might help reduce the incidence of implant failure (only one case [2\%]) and revision surgery.

The solid fusion rates in previous studies investigating lumbar fusion surgery for patients with hemodialysis were $60 \%-79 \%$ [9-12,29], which were quite low (Table 2 ), whereas that in our study was $84 \%$ despite the 2 -year follow-up period. In dynamization-PLIF, even if the cage starts sinking because of vertebral endplate disruption, the pedicle screws can slide parallel to the rod, allowing impaction and compression of the vertebral body and cage frequently. As with intramedullary nailing systems using dynamization for tibia or femur fracture [30,31], the postoperative impaction and compression of fixed segments after dynamization-PLIF would promote solid fusion, a high rate of fusion, and a low rate of hardware failure.

Of note, our study has several limitations. First, the

Table 2. Summary of previous reports of hemodialysis patients who underwent lumbar spinal fusion surgery

\begin{tabular}{|c|c|c|c|c|c|c|}
\hline Authors \& year & $\begin{array}{l}\text { Patient characteristics, } \\
\text { surgical procedure }\end{array}$ & $\begin{array}{l}\text { No. of } \\
\text { patients }\end{array}$ & $\begin{array}{l}\text { Fusion } \\
\text { rate }(\%)\end{array}$ & Fusion definition & $\begin{array}{l}\text { Reoperation rate for } \\
\text { mechanical failure }(\%)\end{array}$ & Details of reoperation \\
\hline Han et al. [9] (2009) & Hemodialysis patients, PLIF & 5 & 60 & Radiographic evaluation & NA & NA \\
\hline Yu et al. [10] (2011) & $\begin{array}{l}\text { Hemodialysis patients, PLF or } \\
\text { PLIF }\end{array}$ & 33 & 75 & Radiographic evaluation & 6.1 & Implant failure 2 cases \\
\hline Chikawa et al. [29] (2013) & $\begin{array}{l}\text { Hemodialysis patients, only } \\
\text { listed as fusion }\end{array}$ & 10 & NA & NA & 10.0 & ASD 1 case \\
\hline Yamada et al. [11] (2016) & $\begin{array}{l}\text { Hemodialysis patients, poste- } \\
\text { rior fusion }\end{array}$ & 14 & 79 & Radiographic evaluation & 21.0 & ASD 3 cases \\
\hline Hori et al. [12] (2019) & $\begin{array}{l}\text { Hemodialysis patients, only } \\
\text { listed as fusion }\end{array}$ & 8 & 63 & Radiographic evaluation & 38.0 & Implant failure 2 cases, ASD 1 case \\
\hline Our series & $\begin{array}{l}\text { Hemodialysis patients, dynam- } \\
\text { ization-PLIF }\end{array}$ & 50 & 84 & Radiographic evaluation & 6.0 & ASD 2 cases, implant failure 1 case \\
\hline
\end{tabular}

PLIF, posterior lumbar interbody fusion; NA, not available; PLF, posterolateral fusion; ASD, adjacent segment disease. 
sample size was quite small. Second, this study is retrospective in design and not comparative. Third, this singlearm study had no control group and patient-reported outcome; thus, the effectiveness of this procedure could not be emphasized. Future efforts, such as comparative studies with conventional PLIF for patients with hemodialysis, including patient-reported outcomes, are necessary to confirm the validity of dynamization-PLIF.

\section{Conclusions}

In patients with hemodialysis who underwent dynamization-PLIF for HSA, lumbar lordosis and local lordosis were improved, the solid fusion rate was favorable (89.3\%), and the reoperation rate within 2 years after surgery was relatively low. Although surgical treatment for lumbar HSA is challenging, dynamization-PLIF can provide favorable surgical outcomes.

\section{Conflict of Interest}

No potential conflict of interest relevant to this article was reported.

\section{Author Contributions}

Taiki Yasukawa wrote and prepared the manuscript, and all of the authors participated in the study design. All authors have read, reviewed, and approved the article.

\section{References}

1. Morimoto S, Nishioka H, Morita T, et al. Characteristics of 20-year survivors undergoing maintenance hemodialysis. Ther Apher Dial 2010;14:547-51.

2. Maruo K, Moriyama T, Tachibana T, et al. Prognosis and adjacent segment disease after lumbar spinal fusion surgery for destructive spondyloarthropathy in long-term hemodialysis patients. J Orthop Sci 2017;22:248-53.

3. Kuntz D, Naveau B, Bardin T, Drueke T, Treves R, Dryll A. Destructive spondylarthropathy in hemodialyzed patients: a new syndrome. Arthritis Rheum 1984;27:369-75.

4. Fiocchi O, Bedani PL, Orzincolo C, et al. Radiological features of dialysis amyloid spondyloarthropathy. Int J Artif Organs 1989;12:216-22.
5. Yamamoto T, Matsuyama Y, Tsuji T, Nakamura H, Yanase M, Ishiguro N. Destructive spondyloarthropathy in hemodialysis patients: comparison between patients with and those without destructive spondyloarthropathy. J Spinal Disord Tech 2005;18:283-5.

6. Ito M, Abumi K, Takeda N, Satoh S, Hasegawa K, Kaneda K. Pathologic features of spinal disorders in patients treated with long-term hemodialysis. Spine (Phila Pa 1976) 1998;23:2127-33.

7. Sebert JL, Fardellone P, Marie A, et al. Destructive spondylarthropathy in hemodialyzed patients: possible role of amyloidosis. Arthritis Rheum 1986;29:3013.

8. Sasaki M, Abekura M, Morris S, et al. Microscopic bilateral decompression through unilateral laminotomy for lumbar canal stenosis in patients undergoing hemodialysis. J Neurosurg Spine 2006;5:494-9.

9. Han IH, Kim KS, Park HC, et al. Spinal surgery in patients with end-stage renal disease undergoing hemodialysis therapy. Spine (Phila Pa 1976) 2009;34:1990-4.

10. Yu YH, Chen WJ, Chen LH, Niu CC, Fu TS, Lai PL. Posterior instrumented lumbar spinal surgery in uremic patients under maintenance hemodialysis. Spine (Phila Pa 1976) 2011;36:660-6.

11. Yamada T, Yoshii T, Hirai T, et al. Clinical outcomes of spinal surgery for patients undergoing hemodialysis. Orthopedics 2016;39:e863-8.

12. Hori Y, Takahashi S, Terai H, et al. Impact of hemodialysis on surgical outcomes and mortality rate after lumbar spine surgery: a matched cohort study. Spine Surg Relat Res 2018;3:151-6.

13. Schnake KJ, Schaeren S, Jeanneret B. Dynamic stabilization in addition to decompression for lumbar spinal stenosis with degenerative spondylolisthesis. Spine (Phila Pa 1976) 2006;31:442-9.

14. Fay LY, Wu JC, Tsai TY, Wu CL, Huang WC, Cheng H. Dynamic stabilization for degenerative spondylolisthesis: evaluation of radiographic and clinical outcomes. Clin Neurol Neurosurg 2013;115:535-41.

15. Kuo CH, Chang PY, Wu JC, et al. Dynamic stabilization for L4-5 spondylolisthesis: comparison with minimally invasive transforaminal lumbar interbody fusion with more than 2 years of follow-up. Neurosurg Focus 2016;40:E3.

16. Yilmaz A, Senturk S, Sasani M, et al. Disc rehydration after dynamic stabilization: a report of 59 cases. 
Asian Spine J 2017;11:348-55.

17. Inose $\mathrm{H}$, Kato $\mathrm{T}$, Yuasa $\mathrm{M}$, et al. Comparison of decompression, decompression plus fusion, and decompression plus stabilization for degenerative spondylolisthesis: a prospective, randomized study. Clin Spine Surg 2018;31:E347-52.

18. Kunogi J, Hasue M. Diagnosis and operative treatment of intraforaminal and extraforaminal nerve root compression. Spine (Phila Pa 1976) 1991;16:1312-20.

19. Jenis LG, An HS. Spine update. Lumbar foraminal stenosis. Spine (Phila Pa 1976) 2000;25:389-94.

20. Brantigan JW, Steffee AD. A carbon fiber implant to aid interbody lumbar fusion: two-year clinical results in the first 26 patients. Spine (Phila Pa 1976) 1993;18:2106-7.

21. Madan S, Boeree NR. Outcome of posterior lumbar interbody fusion versus posterolateral fusion for spondylolytic spondylolisthesis. Spine (Phila Pa 1976) 2002;27:1536-42.

22. Fogel GR, Toohey JS, Neidre A, Brantigan JW. Fusion assessment of posterior lumbar interbody fusion using radiolucent cages: $\mathrm{X}$-ray films and helical computed tomography scans compared with surgical exploration of fusion. Spine J 2008;8:570-7.

23. Chikuda H, Yasunaga H, Horiguchi $H$, et al. Mortality and morbidity in dialysis-dependent patients undergoing spinal surgery: analysis of a national administrative database in Japan. J Bone Joint Surg Am 2012;94:433-8.
24. Chou D, Lau D, Skelly A, Ecker E. Dynamic stabilization versus fusion for treatment of degenerative spine conditions. Evid Based Spine Care J 2011;2:33-42.

25. Guigui P, Ferrero E. Surgical treatment of degenerative spondylolisthesis. Orthop Traumatol Surg Res 2017;103:S11-20.

26. Hoppe S, Schwarzenbach O, Aghayev E, Bonel H, Berlemann U. Long-term outcome after monosegmental L4/5 stabilization for degenerative spondylolisthesis with the Dynesys device. Clin Spine Surg 2016;29:72-7.

27. Ohtonari T, Nishihara N, Suwa K, Ota T, Koyama T. Dynamic stabilization for degenerative spondylolisthesis and lumbar spinal instability. Neurol Med Chir (Tokyo) 2014;54:698-706.

28. Okamoto N, Kawamura N, Kunogi J. Two year follow-up outcomes of dynamization-PLIF for destructive spondyloarthropathy. J Spine Res 2014;5:1020-5.

29. Chikawa T, Sakai T, Bhatia NN, et al. Clinical outcomes of spinal surgery in patients treated with hemodialysis. J Spinal Disord Tech 2013;26:321-4.

30. Perumal R, Shankar V, Basha R, Jayaramaraju D, Rajasekaran S. Is nail dynamization beneficial after twelve weeks: an analysis of 37 cases. J Clin Orthop Trauma 2018;9:322-6.

31. Wu CC. The effect of dynamization on slowing the healing of femur shaft fractures after interlocking nailing. J Trauma 1997;43:263-7. 